\title{
N $88-14334$
}

Spectral and radiometric calibration of the Airborne Visible/Infrared Imaging Spectrometer

Gregg Vane, Thomas G. Chrien, Edward A. Miller, John H. Reimer

Jet Propulsion Laboratory, California Institute of Technology 4800 Oak Grove Drive, Pasadena, California 91109

\begin{abstract}
The laboratory spectral and radiometric calibration of the Airborne Visible/Infrared Imaging Spectrometer (AVIRIS) used in the radiometric calibration of all AVIRIS science data collected in 1987 is described. The instrumentation and procedures used in the calibration are discussed and the calibration accuracy achieved in the laboratory as determined by measurement and calculation is compared with the calibration requirements. Instrument performance factors affecting radiometry are described. The paper concludes with a discussion of future plans.
\end{abstract}

\subsection{INTRODUCTION}

AVIRIS is a second-generation imaging spectrometer developed at the Jet Propulsion Laboratory for use in earth remote sensing studies across a broad spectrum of scientific disciplines, including botany, geology, hydrology and oceanography. ${ }^{1}$ Construction and calibration of the sensor was completed in June 1987. Data were collected for 20 investigators between June and October 1987. The calibration described in this paper was the one used to radiometrically calibrate all AVIRIS science data collected in 1987.

AVIRIS acquires images in 220 contiguous 10-nanometer ( $\mathrm{nm}$ ) spectral bands in the region between 0.40 and $2.45 \mathrm{micrometers}(\mu \mathrm{m})$. The instantaneous field of view of AVIRIS is 1 milliradian and the field of view as defined by the scan angle is 30 degrees. This results in images covering a $10.5 \mathrm{~km}$ swath composed of picture elements (pixels) which subtend $20 \mathrm{~m}$ on the ground from the $20 \mathrm{~km}$ altitude of the NASA U-2. AVIRIS gathers spectral images in the whisk-broom imaging mode, employing foreoptics with a scan mirror which scans in one direction, then rapidly returns to the start position for the next scan line. The scan rate and detector readout timing were designed to provide a 17 percent spatial oversampling at sea level in both the cross-track and along-track dimensions. This results in the acquisition of 614 pixels in each scan line. Geometric rectification removes the oversampling, resulting in an image 550 pixels across. Twelve scans are completed per second. During the fly-back portion of the scan cycle, a shutter closes the foreoptics from the rest of the optical system while a detector dark current measurement is made.

The foreoptics are connected to four spectrometers with optical fibers. Spectrometer A contains a 32-element line array of silicon detectors and spectrometers B, C, and D each employ 64-element line arrays of indium antimonide detectors. While a total of 224 spectral bands are actually acquired, the readout architecture of the detector arrays renders the first band of each array unuseable; there are thus 220 useable raw spectral bands. The useable spectral ranges of spectrometers A, B, C, and D are 0.40 to $0.71,0.68$ to $1.28,1.24$ to 1.86 , and 1.83 to $2.45 \mu \mathrm{m}$, respectively, as currently aligned. During radiometric calibration the spectral region covered by the 220 raw spectral bands is resampled to remove overlap between spectrometers and equalize the spectral sampling interval across the entire 0.40 to $2.45 \mu \mathrm{m}$ region. This results in 210 spectral bands over this region in the radiometrically corrected data.

Each spectrometer additionally receives input via a second optical fiber from an onboard calibration source consisting of a tungsten lamp, a four-position filter wheel containing two neutral density filters, a didymium oxide filter and an opaque position. The neutral density filters provide broad-spectral-band energy at two intensity levels to monitor radiometric stability. The didymium oxide filter provides several spectral absorption features throughout each of the spectral regions of the four AVIRIS spectrometers to monitor spectral calibration. The opaque position is used in conjunction with the shutter in the foreoptics to provide a measurement of dark current. Complete details of the sensor can be found in the papers in these Proceedings. 2

Work with the predecessor to AVIRIS, the Airborne Imaging Spectrometer (AIS), which was an engineering test bed sensor for infrared area detector arrays, has shown clearly the power of imaging spectroscopy as a tool for studying the earth. 3,4 The ability to acquire a complete, high resolution spectrum for each pixel in the scene has made possible from an airborne platform the direct identification of minerals and the assessment of the health and biochemical composition of plants. The data also contain spectral features arising from $\mathrm{CO}_{2}$ and water vapor in the atmosphere; such data are of use in compensating for atmospheric absorption. To derive the maximum information from such data, however, it is critical that the instrument be well-calibrated spectrally and radiometrically. Work with AIS and with laboratory and field spectroradiometers 5,6 provided guidance in establishing the calibration requirements for AVIRIS, which are summarized in Table 1. Table 1 also includes the achieved calibration performance as determined through measurement and calculation.

Table 1. Spectral and Radiometric Calibration Requirements and Achieved Performance

\begin{tabular}{ccc}
\hline \multicolumn{1}{c}{ Parameter } & Required & Achieved \\
\hline Spectral Calibration & $5 \mathrm{~nm}$ & $2.1 \mathrm{~nm}$ \\
Absolute Radiometry & $10 \%$ & $7.3 \%$ \\
Band-to-Band Radiometry & $0.5 \%$ & $0.4 \%$ \\
\hline
\end{tabular}


The requirement for spectral calibration derives from the need to accurately determine the wavelength positions of diagnostic spectral features in earth surface materials. The requirement for relative radiometric calibration, as defined by the spectral band-to-band calibration required to remove the effects of the non-uniformity of response of the detectors, derives from the need to accurately assess the relative strengths of spectral features. The requirement for absolute radiometric calibration was driven by the desire to be able to compare multitemporal data sets of dynamic earth surface materials such as plants and water to assess changes in reflectance over time, and by the need in some applications to determine the absolute magnitudes of various spectral features. Also, theoretical models of atmospheric scattering and absorption require absolutely calibrated data for their successful interpretation.

In the course of calibrating AVIRIS in the laboratory prior to the $1987 \mathrm{flight}$ season, all of the calibration requirements were met. In the following pages, we discuss the details of the procedures used and summarize the results of each step of the calibration.

\subsection{SPECTRAL CALIBRATION}

The purpose of the spectral calibration of AVIRIS is threefold: (1) To map the wavelength distribution of light falling on the detector elements to within $5 \mathrm{~nm},(2)$ to determine the wavelength bandwidth falling on each detector element, and (3) to determine the spectral sampling interval of each spectrometer as defined by the spacing between the center wavelengths of each spectral band. The steps in performing this work are: (1) Calibrating a laboratory monochromator, (2) aligning the monochromator with AVIRIS, and (3) recording the scan of a narrow spectral bandwidth of light from the monochromator across the AVIRIS detectors.

\subsection{Monochromator calibration}

A Jarrell-Ash model 82-487 laboratory monochromator is used in the spectral calibration of AVIRIS. By choosing the widths of the entrance and exit slits, the spectral bandwidth of the monochromator can be tailored as desired. In order to cover the full wavelength range of AVIRIS, three gratings are alternately employed. Wavelength output of the laboratory monochromator is controlled by the angular position of the grating, which is provided by a worm and gear drive attached to a position counter.

Calibration of the monochromator is performed with a mercury pen lamp, using multiple orders of the emission line at $546.1 \mathrm{~nm}$. A plot of the monochromator counter reading as a function of order times $546.1 \mathrm{~nm}$ is used to determine the relationship between the counter reading and wavelength. Figure 1 shows such a plot for the monochromator. The circles on the plot represent the observed counter

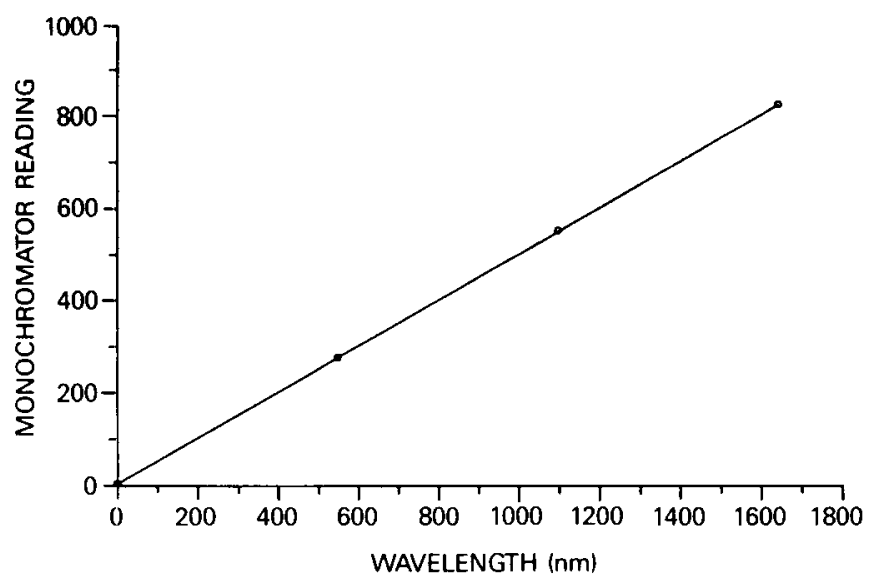

Figure 1. Plot of monochromator reading as a function of wavelength. The straight line is a least squares fit to the multiple orders of the $546.1 \mathrm{~nm}$ mercury line.

readings for zero, first, second, and third orders of the mercury emission line. The straight line connecting the circles is a least squares linear fit to the data. The fit line passes within $1 \mathrm{~nm}$ of each data point. The slope and y intercept of the plot are used to determine the calibration factor necessary to convert the counter number to the output wavelength. In calibrating AVIRIS for the 1987 flight season, the monochromator counter reading was taken to be a linear function of output wavelength. The error inherent in making this assumption is included in the calibration error budget.

\subsection{AVIRIS spectral calibration procedure}

The linearity of spectral dispersion of each AVIRIS spectrometer is determined before it is integrated with the AVIRIS foreoptics. The output of the monochromator is focussed onto a test optical fiber similar to the one connecting the spectrometer and foreoptics, filling the full numerical aperture of the fiber. The wavelength of the monochromator is scanned at a spectral bandwidth of $1 \mathrm{~nm}$. The monochromator counter reading is noted at the peak signal level from each detector element as measured on an oscilloscope. The counter reading is then converted to wavelength and plotted against the spectral band number. A least squares fit of the center wavelength versus the spectral band number is performed on the data and the deviation of each point from that fit is measured. A plot of wavelength versus spectral band number for the first spectrometer in AVIRIS is shown in Figure 2a. Figure $2 b$ shows a plot of the deviation of each spectral band from the least squares fit. Similar plots were made for the other three AVIRIS spectrometers. The standard deviation of the departure from the least squares fit was $0.71,0.60,0.58$, and $1.84 \mathrm{~nm}$ for spectrometers $\mathrm{A}, \mathrm{B}, \mathrm{C}$, and $\mathrm{D}$, respectively. 

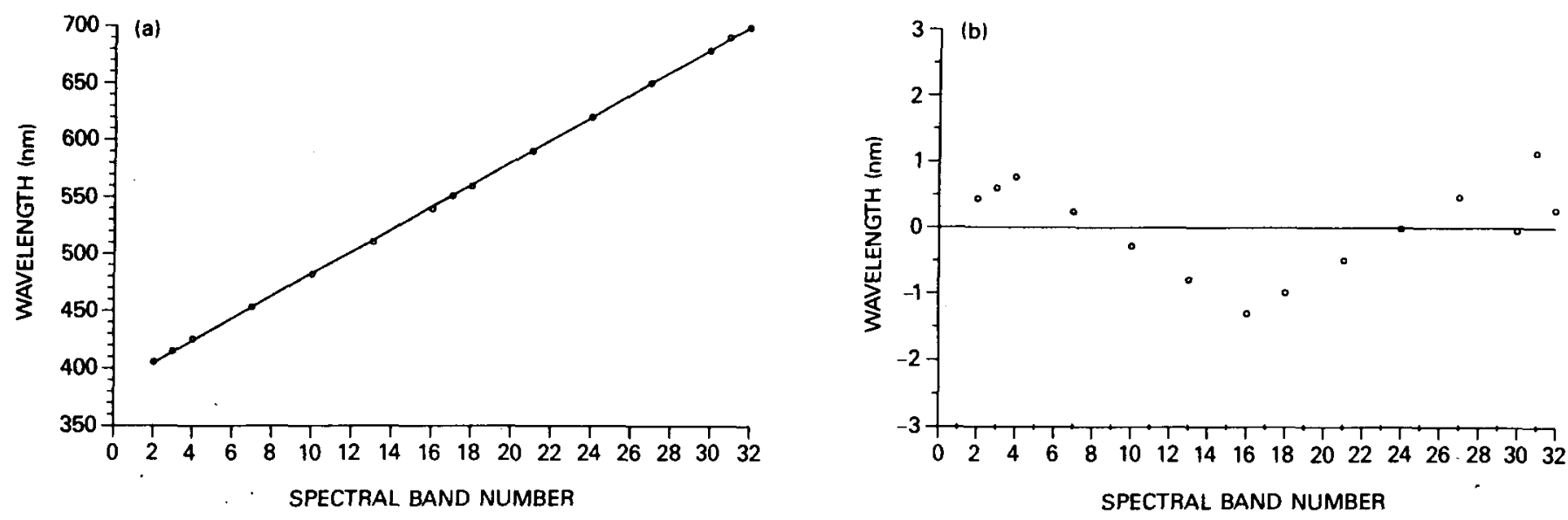

Figure 2. (a) Wavelength versus spectral band number for spectrometer $A$. The straight line is a least squares fit to the measured data. (b) Deviation of the wavelength position of each spectral band versus the least squares fit.

The laboratory setup for performing the spectral calibration of AVIRIS is shown schematically in Figure 3. The calibration is performed after the spectrometers have been integrated with the foreoptics. A tungsten light source is imaged onto the entrance slit of the monochromator. The exit slit of the monochromator is placed at the focus of a 40 inch effective focal length Space Optics Research Lab (SORL) 8 inch aperture collimator. The collimator is centered and aligned so that the full aperture of the AVIRIS foreoptics is filled. In this arrangement, AVIRIS is suspended on a gantry above the collimator. Several spectral bands are chosen for each spectrometer to give a representative sample at the ends and middle of the spectral ranges of the spectrometers. For a given AVIRIS detector element, monochromatic light of $1 \mathrm{~nm}$ bandwidth at increasing wavelength is put into the instrument and the output signal level from that spectral band is recorded as a function of monochromator wavelength, using the AVIRIS ground support computer. A typical plot from spectral band 9, Spectrometer A, is shown in Figure 4. Analysis of this plot shows that the peak signal occurs at $475 \mathrm{~nm}$, which is taken as the center wavelength of the spectral band defined by that detector element. The full spectral width at half the maximum signal from the plot is $9.6 \mathrm{~nm}$, which is taken to be the wavelength bandwidth of AVIRIS spectral band 9 .

The spectral sampling interval, which is defined as the spacing between the center wavelengths of the individual spectral bands, is determined by fitting a straight line to the several spectral bands mapped in each spectrometer according to the method outlined above. The slope of this line is the spectral sampling interval and is used to extrapolate the center wavelengths of the remaining spectral bands in the spectrometer. This approach is employed rather than individually mapping each spectral band because the dispersion of each spectrometer is linear to at least $1.84 \mathrm{~nm}$ over its spectral range. The small error resulting from using this assumption is incorporated into the cnectral calibration error budget.

\subsection{Results of the spectral calibration}

Table 2 summarizes the average spectral bandwidth, the spectral sampling interval, the standard deviation of the linearity measurement, the monochromator calibration accuracy, and the AVIRIS spectral calibration accuracy for each spectrometer. The average bandwidth was

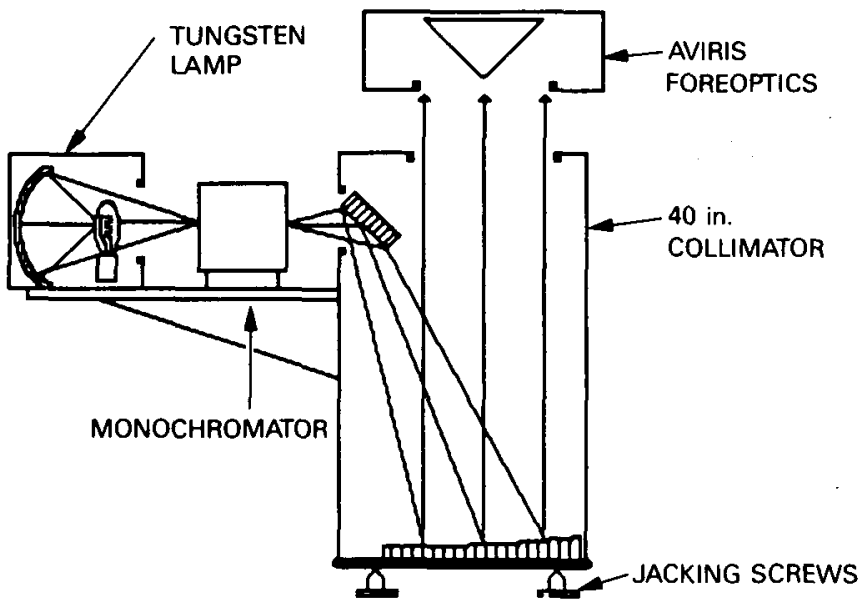

Figure 3. Laboratory setup used in performing the spectral calibration of AVIRIS.

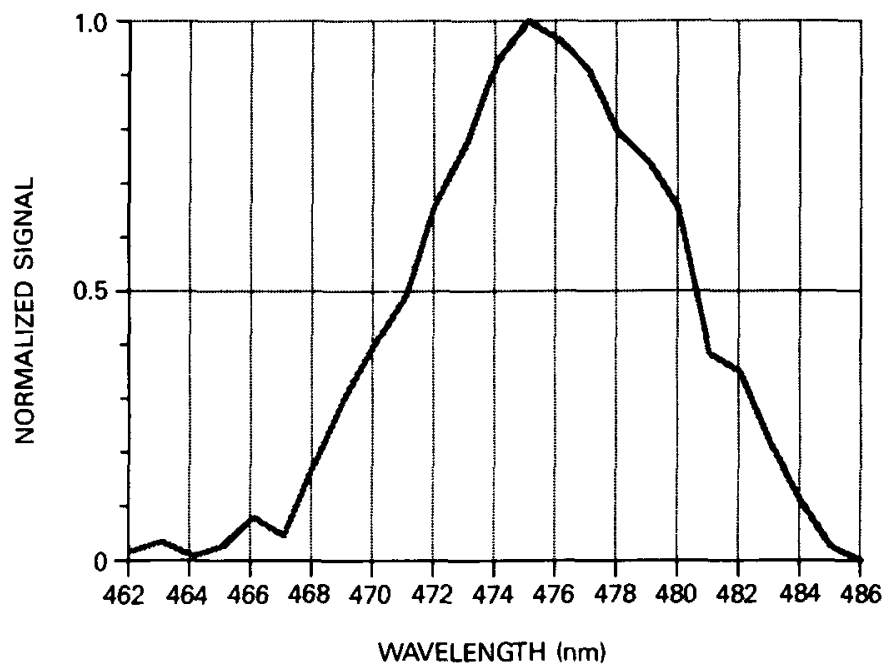

Figure 4. Spectral response curve for spectral band 9 in spectrometer A. 
calculated by averaging the several spectral bandwidths measured in each spectrometer using the narrow band monochromatic light scan technique described earlier. The spectral sampling interval was calculated from the slope of the line passing through those data. The standard deviation of the linearity measurement represents the magnitude of the error introduced by assuming linear dispersion. The monochromator calibration accuracy was measured by performing multiple calibrations with the mercury emission line source and noting the repeatability. The AVIRIS spectral calibration accuracy was then calculated by combining the latter two quantities in Table 2 , derived from measurements in a root sum square fashion. The resulting spectral calibration accuracies exceed the requirements.

Table 2. AVIRIS Spectral Calibration

\begin{tabular}{|c|c|c|c|c|}
\hline \multirow[t]{2}{*}{ Parameter (in units of $\mathrm{nm}$ ) } & \multicolumn{4}{|c|}{ Spectrometer } \\
\hline & A & B & $\mathrm{C}$ & $\mathrm{D}$ \\
\hline Average Bandwidth (FWHM) & 9.7 & 9.7 & 9.0 & 11.6 \\
\hline Spectral Sampling Interval & 10.0 & 9.58 & 9.86 & 9.85 \\
\hline Std. Dev. of Linearity Measurement & 0.71 & 0.60 & 0.58 & 1.84 \\
\hline Monochromator Calibration Uncertainty & 0.5 & 0.5 & 0.5 & 1.0 \\
\hline AVIRIS Spectral Calibration Accuracy & 0.9 & 0.8 & 0.8 & 2.1 \\
\hline
\end{tabular}

\subsection{RADIOMETRIC CALIBRATION}

Radiometric calibration of AVIRIS involves four steps. The first is the calibration of a spectroradiometer against a standard lamp traceable to a National Bureau of Standards (NBS) lamp. The second step is the calibration of the AVIRIS radiance source, a 40-inchdiameter integrating sphere, with the spectroradiometer. Third is the acquisition with AVIRIS of a file of digital numbers (DNs) of the integrating sphere output. Finally, using the spectral calibration, the light transfer curves (radiance versus wavelength) from the integrating sphere, and the DN output acquired with AVIRIS of the sphere, a table of multipliers is constructed which is the calibration file that is applied to all subsequent AVIRIS data to convert the raw instrument DN to radiance. Each of the steps involved in the construction of the calibration file has an error budget associated with it which determines the absolute and relative radiometric calibration accuracies achievable. In the remainder of this section, we describe the steps leading up to the application of the calibration file to the AVIRIS data. In the next section of the paper, we present an analysis of the radiometric calibration accuracy.

\subsection{Calibration of the spectroradiometer}

An Optronics Laboratories model 746 spectroradiometer system is used for radiometric calibration of the AVIRIS integrating sphere. The spectroradiometer is equipped with a silicon detector for use in the 0.4 to $1.1 \mu \mathrm{m}$ region and a lead sulfide detector with a thermal electric cooler for use over the 0.9 to $2.5 \mu \mathrm{m}$ region. The system also includes a monochromator with an integrated chopper, a matched set of gratings and filters, a digital grating drive controller, a detector preamplifier, and a lock-in radiometer. In addition, a 2 inch diameter telescope with a 1.5 degree field of view is used to measure a portion of the integrating sphere surface area comparable to that viewed by AVIRIS. Calibration of the spectroradiometer is done with the telescope in place. During calibration, the entire system is controlled using an MS-DOS compatible computer which automates the processing of data collection and recording. The computer is also used for data reduction.

The laboratory setup used is shown in Figure 5. The spectroradiometer system is positioned on a computer-controlled rotating stage to alternately view a freshly prepared 6 inch square pressed halon target at a 45 degree angle normal to its surface, and the AVIRIS integrating sphere. The pressed halon target is used to calibrate the spectroradiometer in units of radiance. A new halon target is made prior to each calibration using the procedure described by Weidner and Hsia.7 Placed between the halon target and the spectroradiometer are baffles designed and positioned to eliminate stray light from entering the spectroradiometer optics. The halon target is illuminated with a tungsten lamp calibrated as a standard of irradiance by Optronics Laboratories against an NBS-calibrated lamp. The calibrated tungsten lamp is powered by an Optronics calibrated constant-current power supply.

A file of spectral radiance values for the standard lamp-halon target-spectroradiometer configuration is calculated using the expression

$$
L(\lambda)=\frac{\mathrm{I}(\lambda) \cos 45^{\circ} \mathrm{R}(\lambda)(50 \mathrm{~cm})^{2}}{\pi(\mathrm{D} \mathrm{cm})^{2}}
$$

where $L(\lambda)$ is the radiance viewed by the spectroradiometer, $I(\lambda)$ is the irradiance of the calibrated lamp as provided by the manufacturer, $R(\lambda)$ is the reflectance of the halon target $7,50 \mathrm{~cm}$ is the distance at which the lamp was calibrated, and $D$ is the distance between the spectroradiometer and the halon target. DNs are collected with the spectroradiometer at the AVIRIS spectral sampling interval by viewing the halon target illuminated by the standard lamp. A calibration file consisting of a set of multipliers is generated which adjusts the DN output of the spectroradiometer at each wavelength to match the corresponding spectral radiance of the illuminated halon target. This file is applied to all subsequent measurements made with the spectroradiometer to correct its DN output to absolute radiance. 


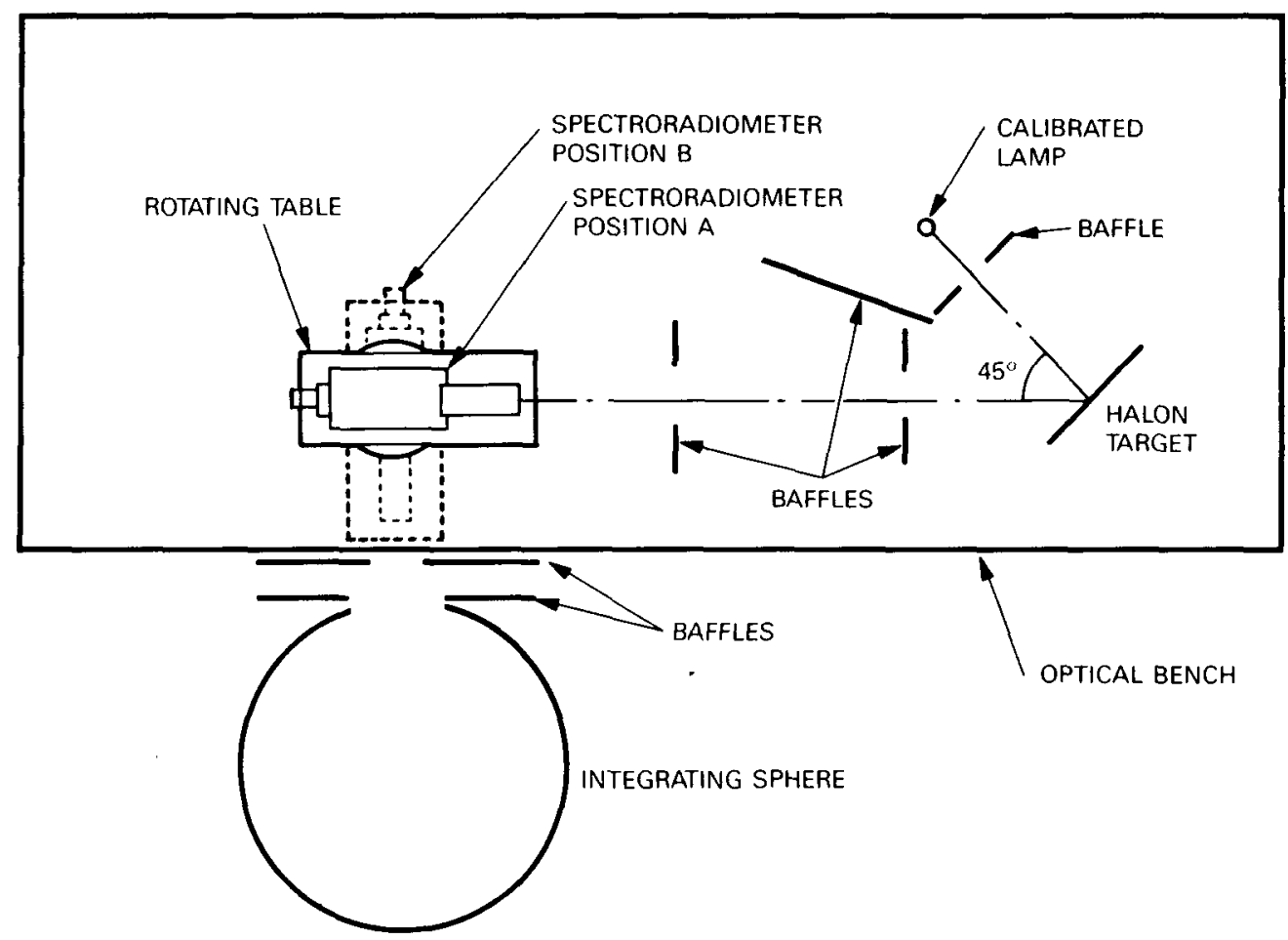

Figure 5. Laboratory setup used in calibrating the AVIRIS spectroradiometer and integrating sphere. All components except the integrating sphere are bolted to an optical bench.

\subsection{Calibration of the integrating sphere}

The radiance source used in the radiometric calibration of AVIRIS is a $40 \mathrm{inch}$ diameter spun aluminum integrating sphere, built by Labsphere Incorporated, with a 16 inch diameter aperture. The interior of the sphere is coated with barium sulfate and is illuminated with four tungsten/halogen 500 watt lamps. The lamps are mounted external to the sphere and cooled with a double ventilating system having a forced-air input and an additional exhaust fan. Each lamp housing and cooling system is isolated from the interior of the sphere by an 8 inch-diameter water-free quartz diffuser. Outside the sphere, between the diffuser and each lamp, is located an aluminum aperture wheel with five sets of holes giving five intensity levels per lamp. The intensity level nomenclature is level $\mathrm{A}$ for highest intensity and level $\mathrm{E}$ for lowest. The four lamp/aperture wheel combinations can be combined to provide a wide range of light intensities within the sphere. A common setting used during AVIRIS calibration is EEEE, which corresponds to each lamp set at the smallest, or E, aperture. The lamps are powered. with an Electronics Measurements Corp. current- and voltage-regulated power supply.

Two criteria are essential for the integrating sphere to be an adequate stimulus for calibrating AVIRIS. First, the radiance must be uniform across the entire 16 inch aperture. The aperture size was chosen to overfill the AVIRIS field of view by 25 percent at the recommended viewing distance of 12 inches from the plane of the aperture. 8 Second, the output of the sphere should be constant with time. Uniformity of radiance across the aperture was measured as a function of angle and as a function of position across the aperture by the manufacturer using a narrow field of view photometer, and at JPL using the Optronics spectroradiometer. The radiance output was found to be uniform to within 1 percent across the aperture for all lamp settings except those using less than all four lamps. The worst case was for illumination with only one lamp; a 2.3 percent loss of radiance was found at the outer 2 inches of the aperture opposite the lamp. This setting is not used in radiometric calibration of AVIRIS. Constancy of output of the sphere was measured by calibrating the sphere 20 consecutive times over a period of four hours, which is approximately the time required to complete an AVIRIS calibration. The radiance output was observed to be constant to within 1.1 percent.

The setup for performing the radiometric calibration of the integrating sphere is shown in Figure 5. After the spectroradiometer has been calibrated against the illuminated halon target, the rotating table is commanded to move the spectroradiometer to a viewing position normal to the plane of the 16 inch aperture. The sphere and spectroradiometer are arranged so that the spectroradiometer views the center of the aperture at a distance of 12 inches from the aperture, duplicating the viewing geometry used in calibrating AVIRIS. Large flat baffles painted Cadillac ${ }^{\mathrm{TM}}$ black are used to minimize stray light reflected between the sphere and AVIRIS. These baffles are used in the same relative positions when viewing the sphere with the spectroradiometer. After a 30 minute warm-up period, the spectroradiometer acquires data from the integrating sphere. These raw DNs are converted to radiance as described above. The results of a typical calibration of the AVIRIS integrating sphere are shown in Figure 6 . This is one of the light transfer curves in radiance versus spectral band number subsequently used to convert AVIRIS DNs to radiance.

\subsection{Calibration of AVIRIS}

AVIRIS, the integrating sphere, and the spectroradiometer are all located in the same laboratory, which is temperature and humidity stabilized. After the sphere has been calibrated, it is moved into position beneath AVIRIS, which is suspended from a gantry. Placed between AVIRIS and the integrating sphere is the hatch window from the U-2. The distance of the window from the AVIRIS scan mirror 


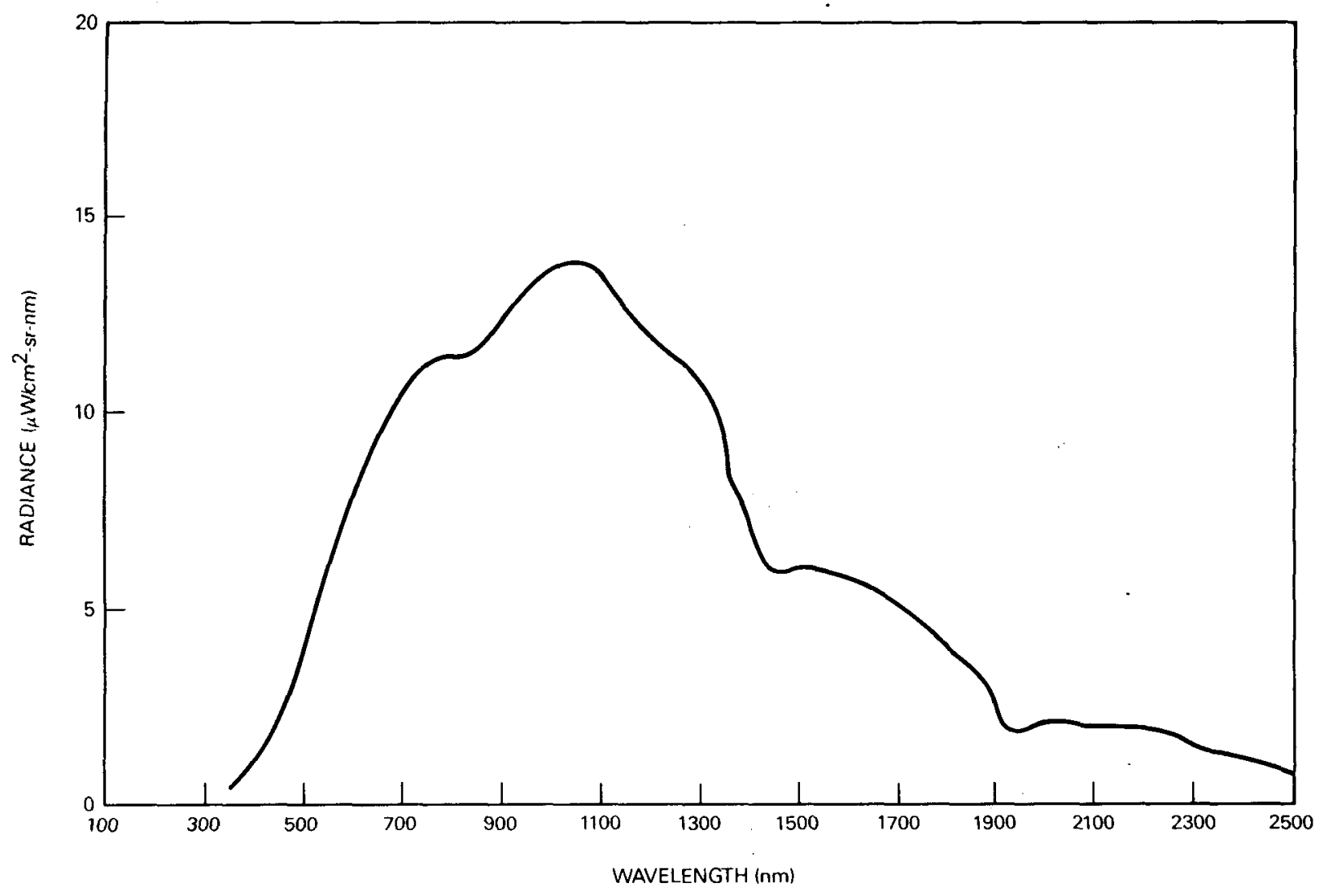

Figure 6. Typical light transfer curve from the AVIRIS integrating sphere at the lowest four-lamp output level (setting EEEE).

is the same as in the airplane. Following the same warm-up procedure used in calibrating the sphere, AVIRIS data are accuired from the sphere at several aperture settings over several hours. During an initial calibration of AVIRIS, data were acquired at 20 sphere intensity settings to determine the linearity of response of AVIRIS. The instrument was found to be linear to within the limits of the measurement technique, which was limited by the thermally induced drift in instrument output discussed in the next section of this paper. In the current instrument configuration, the AVIRIS response was determined to be linear to within 2.4 percent. This allows an adequate radiometric calibration to be performed using less than the full suite of 20 integrating sphere output settings. Two settings have been chosen which, in combination, optimize the signal levels in AVIRIS across the 0.40 to $2.45 \mu \mathrm{m}$ range. At each of these settings, 20 consecutive sets of data are acquired at five minute intervals. Each data set consists of 10 seconds or 120 scan lines of data, with each scan line containing 614 readings of the sphere output across the 30 degree scan angle of AVIRIS. The mean values of 100 of the scan lines of data are obtained for each run at each wavelength, and the mean of the 20 runs at each of the two integrating sphere output levels is obtained, from which the calibration file is constructed. The details of this process follow.

\section{$\underline{3.4 \text { Creation of the calibration file }}$}

The calibration file used to convert AVIRIS DN values into radiance values is a set of multipliers consisting of 32-bit floating point numbers generated from the laboratory calibration data. These data consist of two components: radiance measurements of the two integrating sphere intensity levels taken with the spectroradiometer, and data taken with AVIRIS while viewing the integrating sphere at the same two intensity levels. These data are decommutated and archived in the same manner as flight data. In generating the calibration file, one of the two integrating sphere intensity settings is chosen for each AVIRIS spectral band based on the criterion of maximum signal without saturation. In generating the calibration file, the data from the one intensity level chosen for the specific spectral band and the zero point on the radiance versus DN plot are used in calculating the multipliers for that spectral band.

The first step in generating the calibration file is subtraction of the dark current from the AVIRIS data. Dark current subtraction is done individually on each of the 100 lines of data using the mean dark current for those 100 lines. For each spectral band the corresponding AVIRIS data are then averaged over time as a function of cross-track sample number. At a given integrating sphere setting, the mean DN value for a given spectral band and cross-track (spatial) sample number is obtained from $2000 \mathrm{DN}$ values (20 sets of data at that integrating sphere output level times 100 samples per data set). This results in a set of $224 \times 614$ mean DN values for the 224 spectral bands and the 614 cross-track pixels. A cross-track dependence is kept in order to account for non-uniform response with scan angle due to vignetting in the foreoptics of AVIRIS. Examples of cross-track plots of DN versus sample number are shown in Figures $7 \mathrm{a}-7 \mathrm{~d}$ for four of the 20 data sets taken at integrating sphere output level EEEE. These examples show that both the mean value and the shape of the cross-track plots vary over time and in a manner that is uncorrelated between spectrometers. This variation enters into the calculation of calibration accuracy discussed in the next section of the paper.

The next step in generating the calibration file is to associate the 614 cross-track mean DN values of every spectral band with the corresponding radiance value for that spectral band, computed by resampling the spectroradiometer measurements to match the spectral 

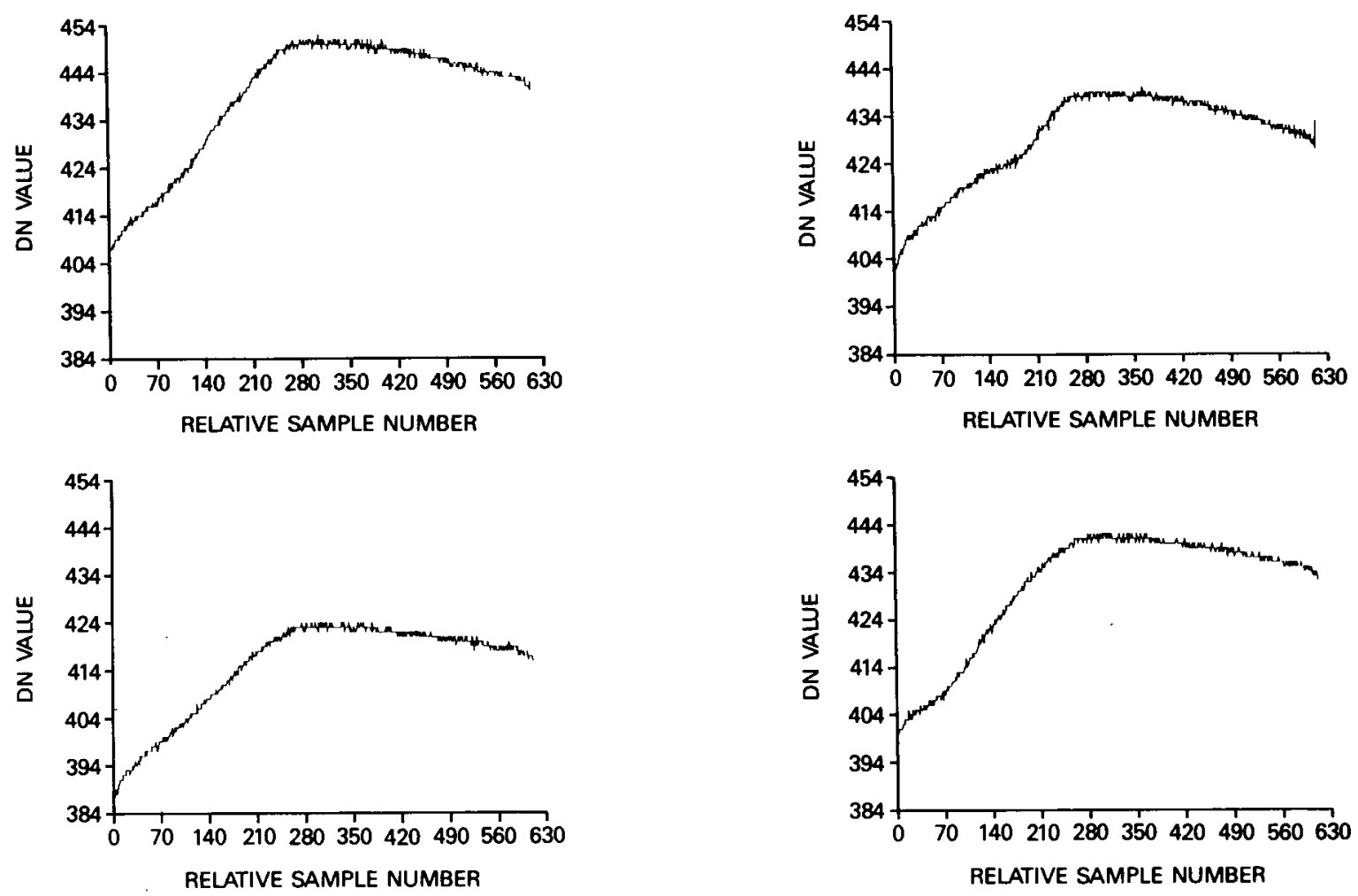

Figure 7. (a) Plots of four sets of raw AVIRIS DN after dark current subtraction versus relative cross-track sample number for spectral band 30, Spectrometer A. Each set of data was acquired from the AVIRIS integrating sphere at output level EEEE.
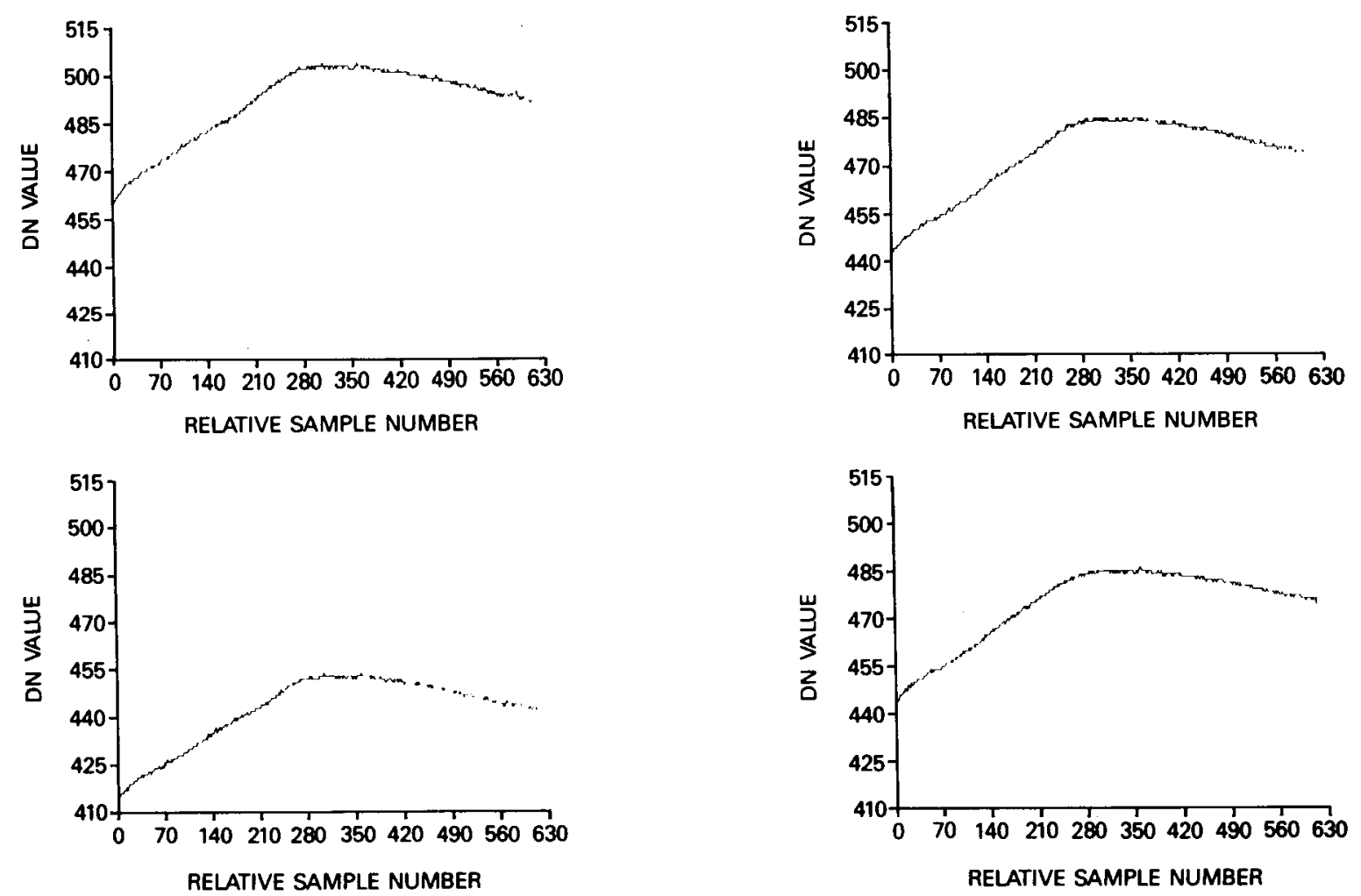

Figure 7. (b) Plots of four sets of raw AVIRIS DN after dark current subtraction versus relative cross-track sample number for spectral band 68 , Spectrometer B. Each set of data was acquired from the AVIRIS integrating sphere at output level EEEE. 

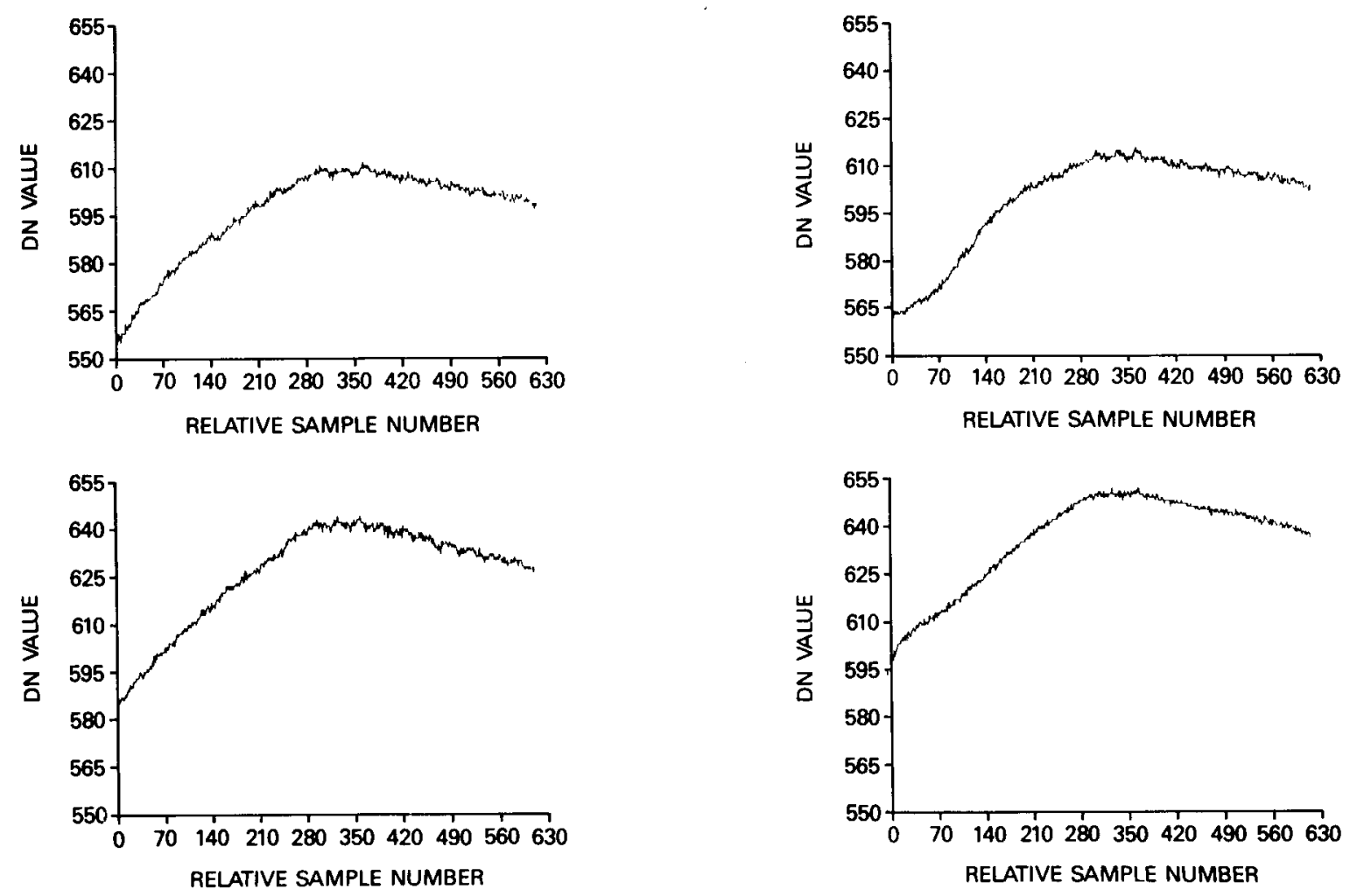

Figure 7. (c) Plots of four sets of raw AVIRIS DN after dark current subtraction versus relative cross-track sample number for spectral band 125, Spectrometer C. Each set of data was acquired from the AVIRIS integrating sphere at output level EEEE.
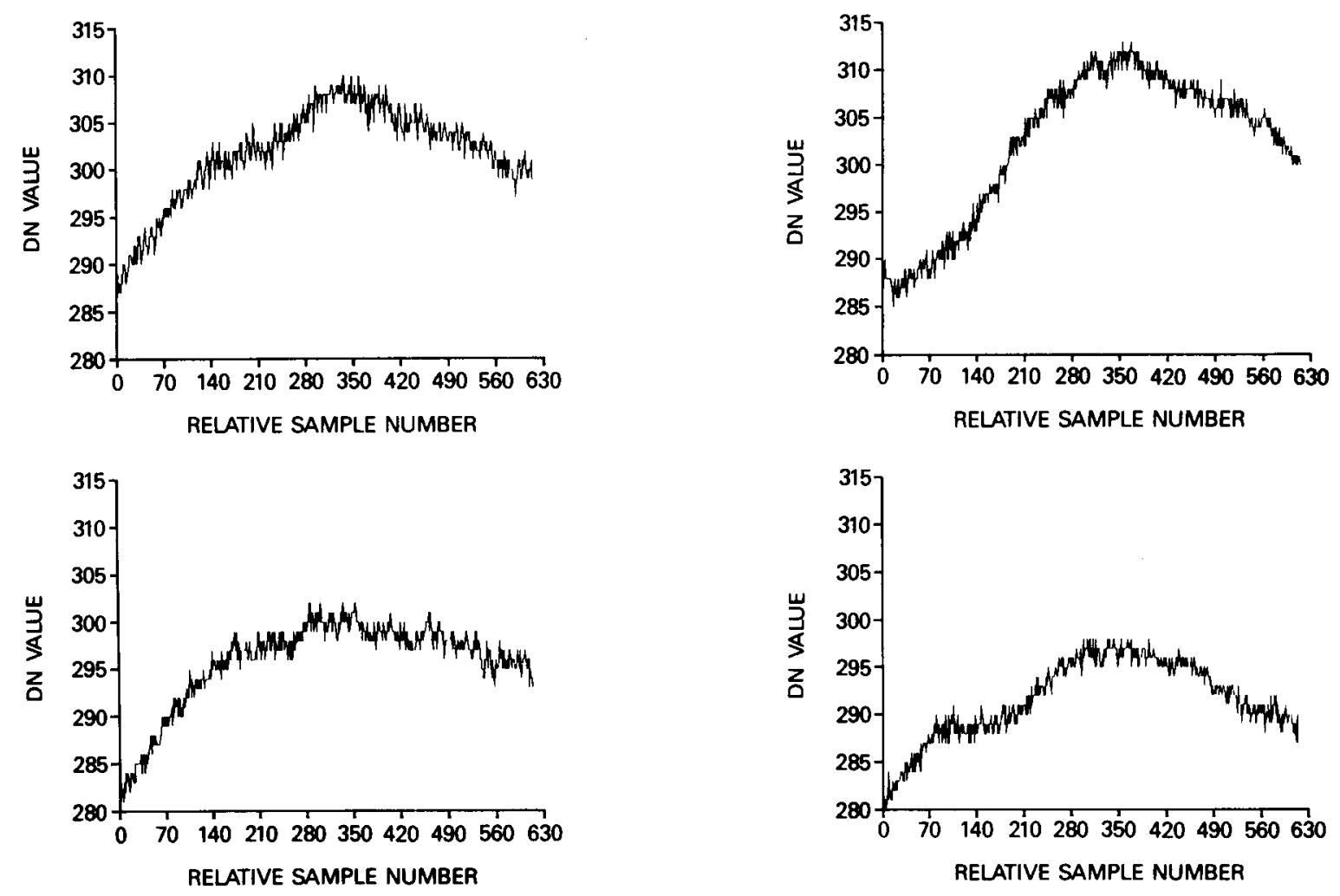

Figure 7. (d) Plots of four sets of raw AVIRIS DN after dark current subtraction versus relative cross-track sample number for spectral band 188, Spectrometer D. Each set of data was acquired from the AVIRIS integrating sphere at output level EEEE. 
spacing of the AVIRIS detector elements. Assuming instrument response linearity, the final set of multipliers is computed as follows:

$$
\operatorname{MULT}(s, b)=\operatorname{RAD}(b) / D N(s, b)
$$

where $s$ is the cross-track sample number, $b$ is the spectral band number, $R A D(b)$ is the radiance of the integrating sphere, and DN(s,b) is the signal level reconded by AVIRIS. This set of $224 \times 614$ multipliers is saved as the calibration file and used in the radiometric calibration of all subsequent AVIRIS data.

\subsection{Application of the calibration file to AVIRIS data}

The radiometric calibration of AVIRIS flight data converts 10 -bit DN values to 32-bit floating point radiance values in terms of $\mu W / \mathrm{cm}^{2}$ $\mathrm{nm}$-sr and linearly scales the values to 16-bit integers. In the process, dark current subtraction, detector equalization, resampling to account for detector readout delays, and reconstruction of the full spectrum accounting for spectral band overlap and varying spectral spacings between spectrometers are performed.

The first step in radiometric calibration processing of flight data is dark current subtraction. One dark current value for each detector element is recorded at the end of each scan line. Dark current subtraction is done using a sliding mean of 101 dark current values as follows:

$$
\mathrm{DN}(\mathrm{s}, \mathrm{b}, \mathrm{L})=\mathrm{DN}(\mathrm{s}, \mathrm{b}, \mathrm{L})-1 / 101 \sum_{\mathrm{k}=\mathrm{L}-50}^{\mathrm{L}+50} \mathrm{DC}(\mathrm{b}, \mathrm{k})
$$

where $s$ is the cross-track sample number, $b$ is the spectral band number, $L$ is the line number, and DC is the dark current.

The next step is detector response equalization. Using the multipliers stored in the calibration file, DN values are converted to 32-bit floating point radiance values, $\mathrm{RAD}(\mathrm{s}, \mathrm{b}, \mathrm{L})$ :

$$
\operatorname{RAD}(s, b, L)=\operatorname{DN}(s, b, L) * \operatorname{MULT}(s, b)
$$

Application of the multipliers at this point should remove the cross-track response asymmetry caused by foreoptics vignetting. Figures 8a - 8d show representative cross-track plots of integrating sphere data before and after this process. These figures show that while there is significant improvement, deviations from an ideal horizontal plot still exist due to time variance in the shape of the cross-track plots. Note the change in scales between the raw and radiometrically calibrated data. The magnitude of the vignetting in the raw data is about $8 \%$. The departure from the uniform cross-track response after calibration is $2 \%$ or less and is time-varying, as discussed in section 4.3 of the paper.

Resampling to correct for detector readout delays is performed next. These delays occur because the detector elements in the line arrays are read sequentially. This results in the last element of an array acquiring a signal from a spot on the ground approximately one pixel further across the scan than was acquired by the first element. Linear interpolation is performed between successive spatial pixels within a scan line as shown in equation 5 . As indicated by this equation, time increases as the sample number decreases. The time between reading out element number 64 of an array and subsequently reading out element number 1 of the same array at the next spatial pixel in the scene is equivalent to two detector element readout periods; thus the number 66 is used in equation 5 . Readout of the 32 element silicon array corresponds in time to readout of elements 1 through 32 within the three 64 element InSb arrays.
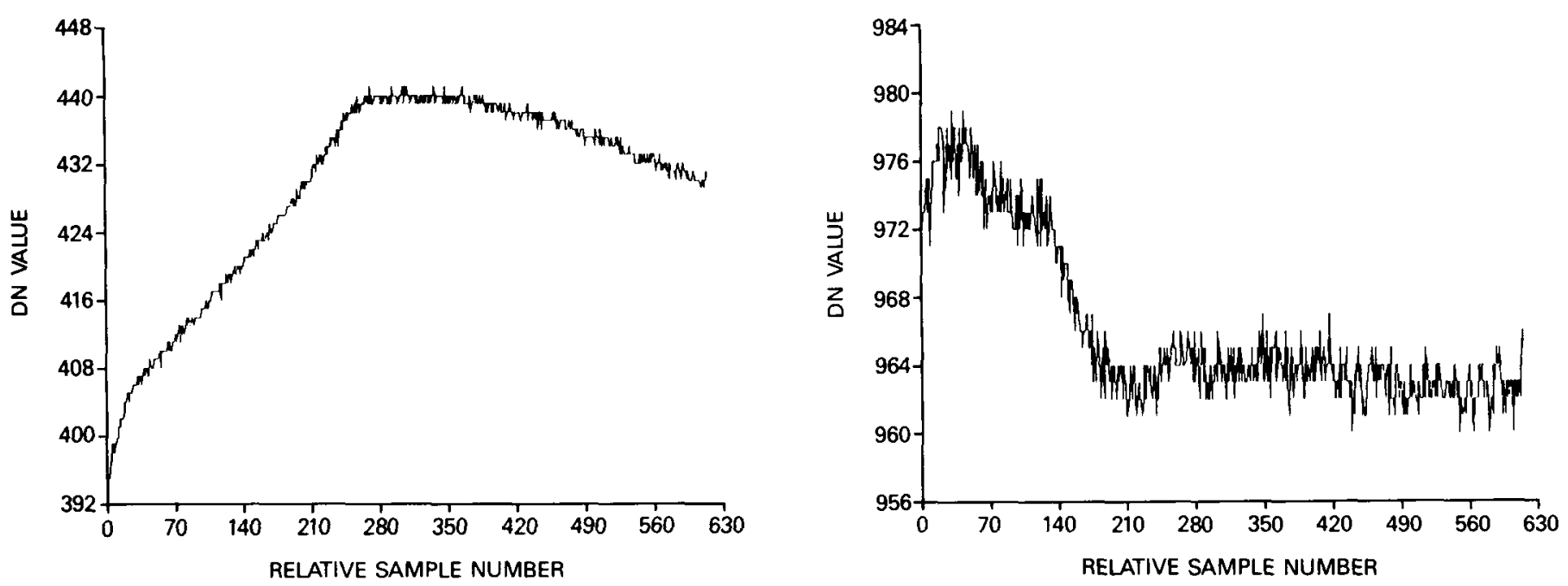

Figure 8. (a) Plots of raw (left) and radiometrically corrected (right) AVIRIS DN versus relative cross-track sample number for spectral band 30 , Spectrometer $A$. Note the difference in scales of the vertical axes of the two plots. 

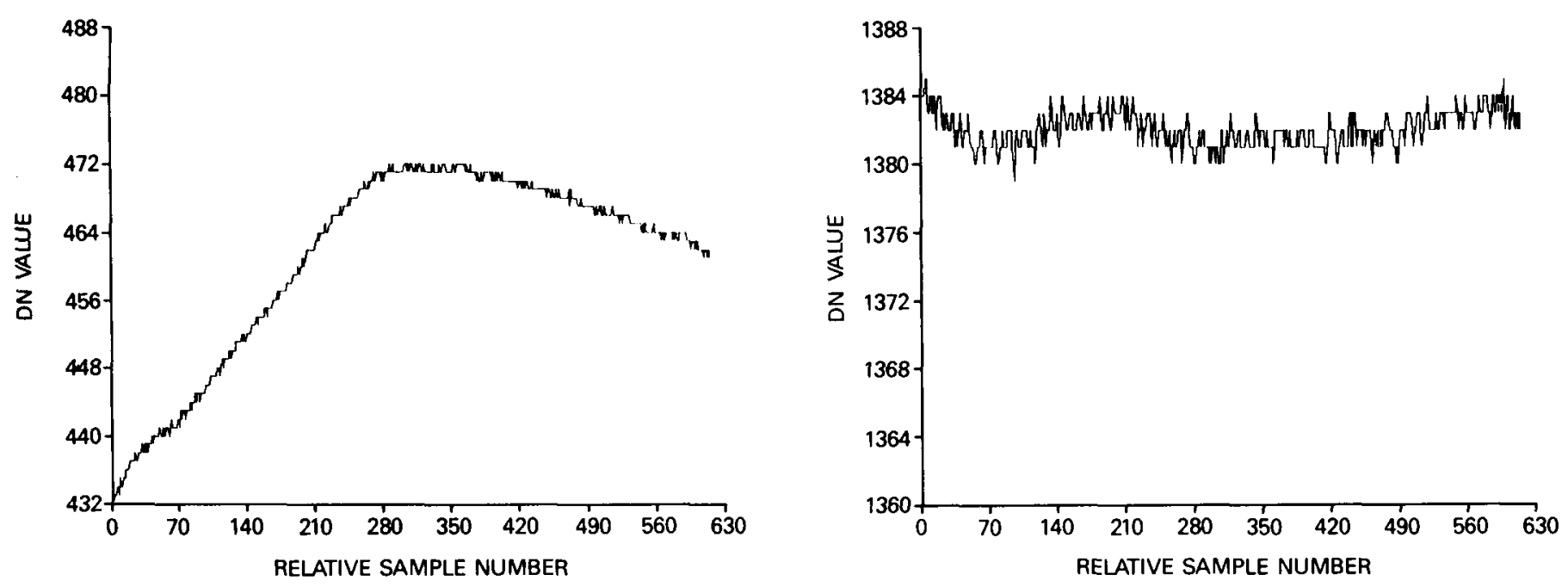

Figure 8. (b) Plots of raw (left) and radiometrically corrected (right) AVIRIS DN versus relative cross-track sample number for spectral band 68 , Spectrometer $B$. Note the difference in scales of the vertical axes of the two plots.
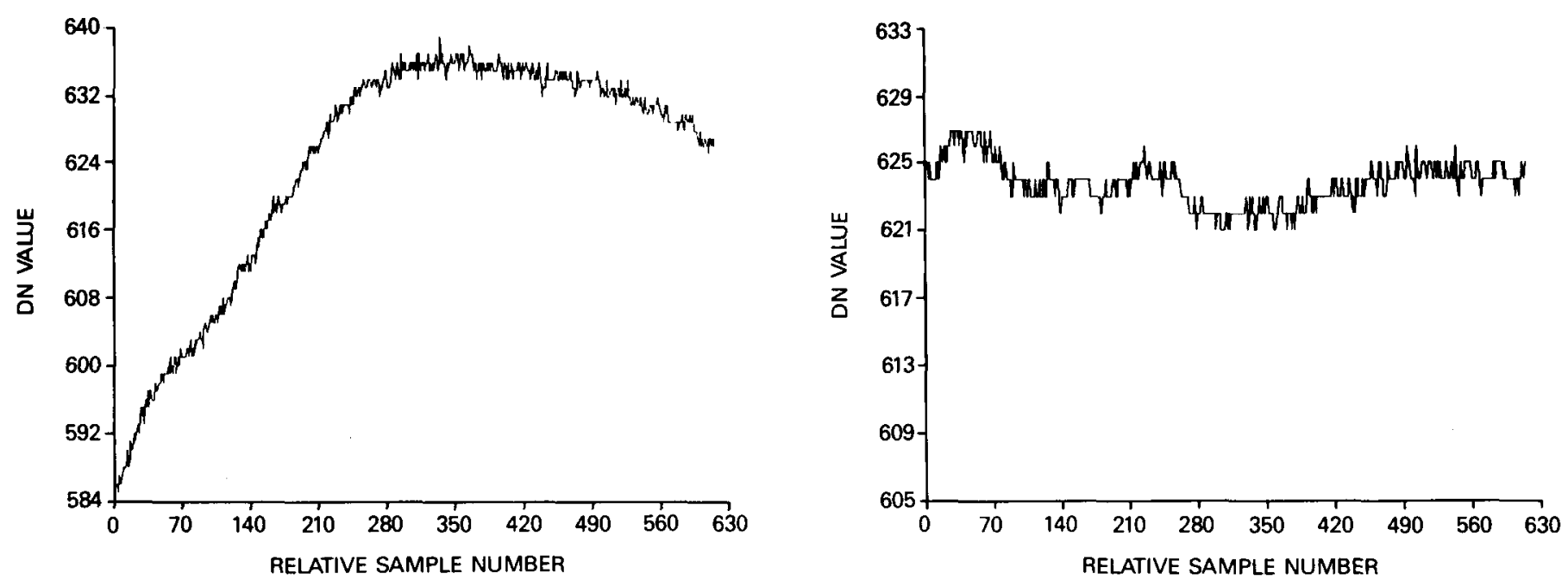

Figure 8. (c) Plots of raw (left) and radiometrically corrected (right) AVIRIS DN versus relative cross-track sample number for spectral band 125 , Spectrometer $C$. Note the difference in scales of the vertical axes of the two plots.
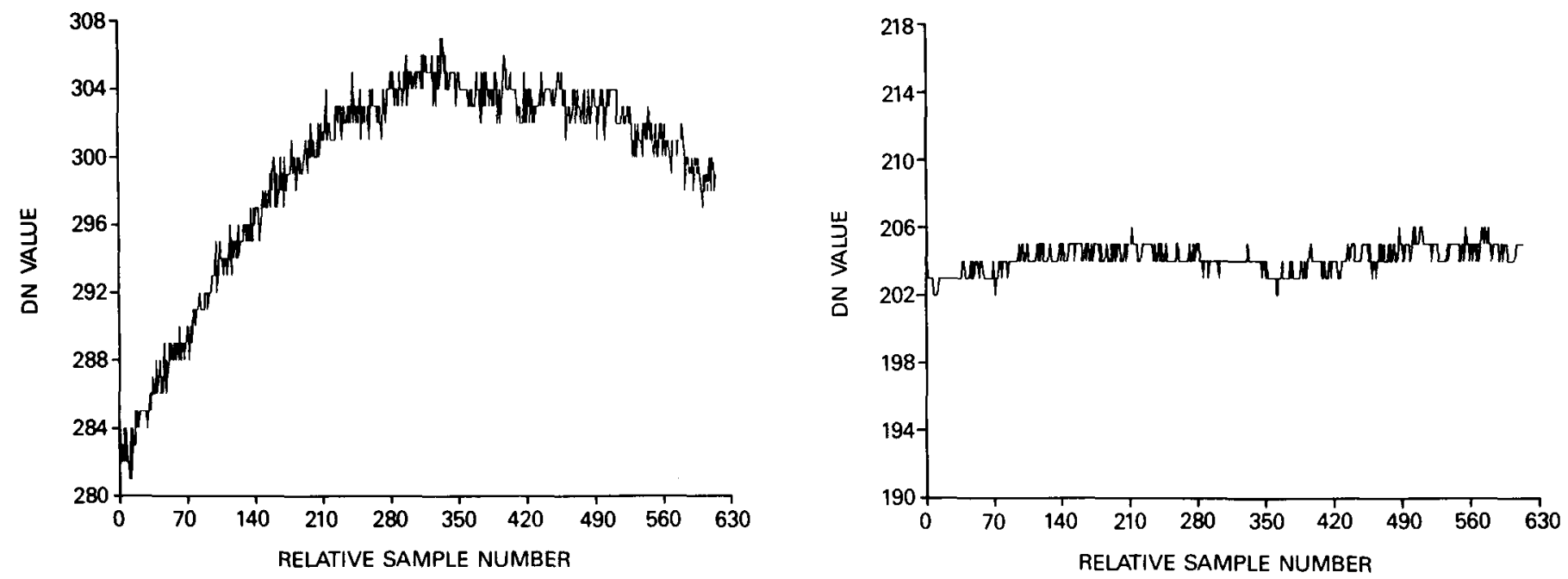

Figure 8. (d) Plots of raw (left) and radiometrically corrected (right) AVIRIS DN versus relative cross-track sample number for spectral band 188, Spectrometer D. Note the difference in scales of the vertical axes of the two plots. 


$$
\operatorname{RAD}^{\prime}(\mathrm{s}, \mathrm{b}, \mathrm{L})=\left[\left(\mathrm{b}^{\prime}-1\right) / 66\right]^{*} \mathrm{RAD}(\mathrm{s}+1, \mathrm{~b}, \mathrm{~L})+\left[\left(67-\mathrm{b}^{\prime}\right) / 66\right]^{*} \mathrm{RAD}(\mathrm{s}, \mathrm{b}, \mathrm{L})
$$

where $b^{\prime}$ is the detector array element number of spectral band $b$.

Following this, the full spectrum is reconstructed. Resampling is performed in the spectral direction to correct for spectral band overlap between spectrometers and to create a spectrum with uniform spacing of spectral band center wavelengths. This step produces 210 spectral bands from 0.4000 to $2.4482 \mu \mathrm{m}$ with $9.8 \mathrm{~nm}$ spectral sampling. The center wavelength falling on each AVIRIS detector is determined during spectral calibration. Ignoring detector element number 1 in each spectrometer, unusable because of the readout architecture of the arrays, each resampled spectral band is computed by linearly interpolating between the two closest spectral bands using equation 6:

$$
\operatorname{RAD}^{\prime \prime}(s, b, L)=\left(\frac{\lambda_{b}-\lambda_{b-}}{\lambda_{b+}-\lambda_{b-}}\right)\left[\operatorname{RAD}^{\prime}(s, b+, L)-R^{\prime} D^{\prime}(s, b-, L)\right]+\operatorname{RAD}^{\prime}(s, b-, L)
$$

where $b$ is the resampled spectral band number, $b$ - is the spectral band nearest to $b$ with wavelength less than $b$, and $b+$ is the spectral band nearest to $b$ with wavelength greater than $b$. Finally, the 32-bit floating point radiance values are converted to 16-bit integers by multiplying each radiance value by a factor of 100 and rounding to the nearest integer value.

As a check of the software and procedure, radiometric rectification was applied to the integrating sphere data used in generating the calibration file. The results are shown in Figure 9. Figure 9a is a spectral plot of the raw AVIRIS data, while Figure $9 \mathrm{~b}$ is a spectral plot of the same data following radiometric rectification. This may be compared to the spectrum of the integrating sphere at the same output intensity shown in Figure 6.
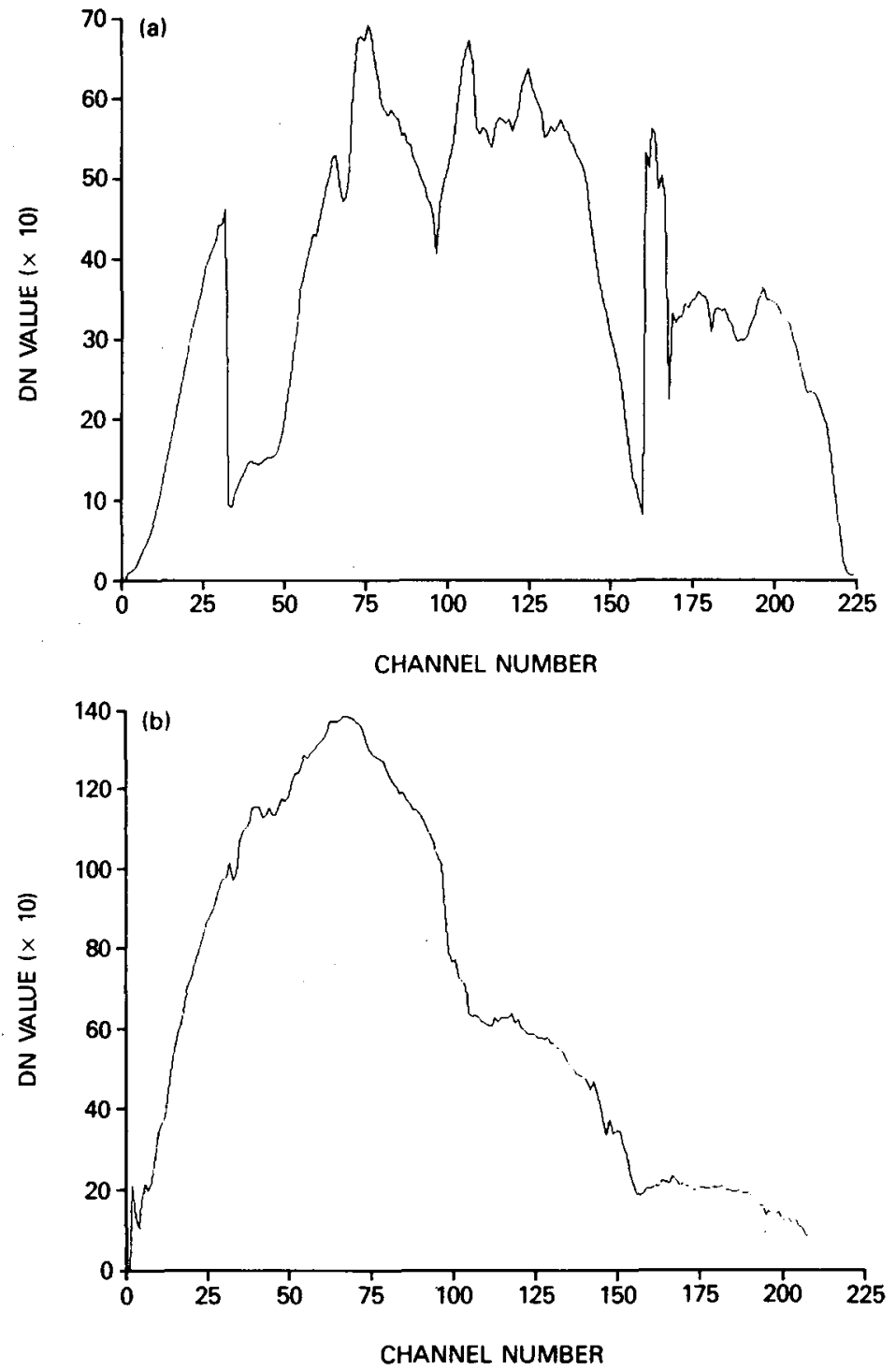

Figure 9. (a) Plot of raw AVIRIS DN after dark current subtraction versus spectral band number from the integrating sphere output level EEEE. (b) Plot of radiometrically corrected AVIRIS data versus spectral band number for the same integrating sphere output level. Compare this plot to the integrating sphere light transfer curve in Figure 6.

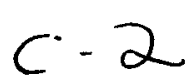




\subsection{RADIOMETRIC CALIBRATION ACCURACY}

The radiometric calibration accuracy of AVIRIS is limited by the accuracy of the integrating sphere calibration, the temporal stability of AVIRIS, the spectral calibration of AVIRIS, and such factors as sensitivity to polarization and to spectral and spatial stray light. Following is a discussion of these factors and a summary of the laboratory radiometric calibration accuracy of the instrument.

\subsection{Accuracy of the integrating sphere calibration}

The accuracy of the integrating sphere calibration was determined by calculating the uncertainty of the transfer from the irradiance of the standard lamp to the radiance of the halon target, measuring the uncertainty in the performance of the spectroradiometer, and measuring the uncertainty in the performance of the integrating sphere. The uncertainty associated with the use of the external irradiance standard and errors associated with its use were calculated using a differential propagation of errors analysis. 9 This calculation included the uncertainty of the calibration of the irradiance standard as provided by Optronics, the uncertainty of the reflectance of the pressed halon target, and the uncertainties introduced by the geometry of the calibration setup. The total uncertainty in the transfer of irradiance to radiance was calculated to be 2.0 percent.

The uncertainty present in the spectroradiometer measurements can be characterized by considering those factors contributing to system drift and the accuracy of the wavelength calibration. Factors contributing to system drift include chopper phase error, detector noise, and optical alignment within the spectroradiometer. These factors are contained in the overall repeatability of the measurements made with the spectroradiometer system. The repeatability was determined by performing a calibration of the spectroradiometer at the beginning and end of the calibration of the integrating sphere and comparing the results with previous calibrations of the spectroradiometer. The mean difference in the before and after calibrations was 1.8 percent. The spectral calibration uncertainty of the spectroradiometer is given by the manufacturer as $1 \mathrm{~nm}$. This has been measured and confirmed at JPL. Spectral calibration uncertainty has its greatest effect on the steepest portion of the light transfer curve for a tungsten lamp, which from Figure 6 is at about $1.4 \mu \mathrm{m}$. A 1 nm uncertainty in spectroradiometer spectral calibration results in a radiance uncertainty of 0.5 percent at that wavelength.

The uncertainty present in the output of the integrating sphere is determined by the accuracy and stability of the lamp power supplies, the degradation with time of the lamps, and the uniformity of radiance across the output aperture of the sphere. The systematic error contributed by the integrating sphere is characterized by the repeatability of its calibration with the spectroradiometer. Multiple calibrations of the integrating sphere over several months, with each calibration consisting of several calibrations during the course of a day, give a repeatability of 1.1 percent, which accounts for power supply accuracy and stability, lamp irradiance drifts, and other unknown error sources. The uniformity of the radiance over the output aperture also affects the integrating sphere calibration accuracy. Work cited earlier gave a worst-case uniformity of 1.0 percent for the lamp and aperture settings used in calibrating AVIRIS.

Table 3 summarizes all the factors affecting the accuracy of the radiance calibration of the AVIRIS integrating sphere. Because these factors are uncorrelated, the contribution from each error source has been combined using the square root of the sum of the squares approach to arrive at a total uncertainty of 3.1 percent.

Table 3. Integrating Sphere Calibration Error Budget

\begin{tabular}{ll}
\hline \multicolumn{1}{c}{ Parameter } & Magnitude (\%) \\
\hline $\begin{array}{l}\text { Irradiance Transfer Uncertainty } \\
\text { Spectroradiometer Stability }\end{array}$ & 2.0 \\
$\begin{array}{l}\text { Spectroradiometer Radiance Calibration } \\
\text { Uncertainty due to Spectral Calibration } \\
\text { Uncertainty of 1 nm } \\
\text { Integrating Sphere Output Stability } \\
\quad \begin{array}{l}\text { Radiance Uniformity Across Integrating } \\
\text { Sphere Output Port }\end{array}\end{array}$ & 1.8 \\
\hline \begin{tabular}{l} 
Integrating Sphere Calibration Accuracy \\
\hline
\end{tabular} & 1.1 \\
\hline
\end{tabular}

\subsection{Temporial stability of AVIRIS response}

The temporal stability was determined from the 20 sets of integrating sphere data acquired during the final calibration of AVIRIS prior to the start of flights aboard the U-2 in June 1987. As described earlier, these data were acquired at 5 minute intervals in an air conditioned laboratory whose temperature was maintained at 68 degrees $F$ to assure the cycling of heaters designed to keep the four spectrometers at constant temperature. The heaters are distributed around the barrel of each spectrometer to maintain constant barrel length and geometry, 
which both affect the alignment between the optical elements. Table 4 shows the results of the stability performance evaluation. Tabulated is the mean dark-current-corrected DN from one spectral band from each of the spectrometers, averaged over the full scan. The mean was constructed from 100 scan lines of data. Runs 7, 11 and 15 are missing due to a tape-read error during decommutation. Below each column of mean values for each run is the mean of all 17 runs, the maximum deviation from the mean, and the ratio of the maximum deviation to the mean. The table shows spectrometer B to have the largest drift in output over the 95 minutes of instrument operation, while spectrometer D has the smallest drift.

Table 4. Spectrometer Stability Versus Time

\begin{tabular}{|c|c|c|c|c|c|}
\hline \multirow{2}{*}{$\begin{array}{c}\text { Run } \\
\text { Number }\end{array}$} & \multirow[b]{2}{*}{ Time (min) } & \multicolumn{4}{|c|}{ Spectrometer (Spectral Band) Signal Level in DN } \\
\hline & & $A(30)$ & B (68) & $C(125)$ & $\mathrm{D}(188)$ \\
\hline 1 & 0 & 439 & 473 & 597 & 301 \\
\hline 2 & 5 & 429 & 492 & 600 & 302 \\
\hline 3 & 10 & 439 & 458 & 607 & 301 \\
\hline 4 & 15 & 438 & 466 & 612 & 299 \\
\hline 5 & 20 & 434 & 450 & 613 & 303 \\
\hline 6 & 25 & 434 & 455 & 619 & 301 \\
\hline 7 & 30 & & & & \\
\hline 8 & 35 & 430 & 447 & 622 & 301 \\
\hline 9 & 40 & 429 & 461 & 624 & 299 \\
\hline 10 & 45 & 416 & 442 & 626 & 296 \\
\hline 11 & 50 & & & & \\
\hline 12 & 55 & 424 & 438 & 632 & 300 \\
\hline 13 & 60 & 421 & 446 & 630 & 296 \\
\hline 14 & 65 & 427 & 474 & 635 & 294 \\
\hline 15 & 70 & & & & \\
\hline 16 & 75 & 431 & 474 & 636 & 292 \\
\hline 17 & 80 & 431 & 440 & 635 & 296 \\
\hline 18 & 85 & 436 & 464 & 640 & 297 \\
\hline 19 & 90 & 436 & 439 & 636 & 298 \\
\hline 20 & 95 & 431 & 458 & 635 & 291 \\
\hline Mean: & & 431 & 457 & 623 & 298 \\
\hline Std De & & 6.34 & 15 & 13.4 & 3.5 \\
\hline Std De & ean: & $1.5 \%$ & $3.3 \%$ & $2.2 \%$ & $1.2 \%$ \\
\hline $\operatorname{Max} D$ & & 15 & 35 & 26 & 7 \\
\hline $\operatorname{Max} D$ & Mean: & $3.5 \%$ & $7.7 \%$ & $4.2 \%$ & $2.4 \%$ \\
\hline
\end{tabular}

Two factors have been identified to account for the drift in output: (1) The spectrometer heaters are causing a distortion in the barrel geometry while they are on the heating cycle. This causes the signal to drift off the detector array in a direction perpendicular to the dispersion, resulting in a drop in signal while the heaters are on, but no shift in spectral alignment. After the heater of a given spectrometer switches off, the temperature of the barrel becomes uniform across the barrel and the geometry returns to optimal, as does the output signal level. (2) The alignment of the spectrometers is also affected by thermally induced warpage of the rails on which the spectrometers are mounted. This effect results in a similar shift of image position on the detector arrays.

There are potentially two ways of correcting for the thermally induced drift in signal output of the spectrometers. The onboard calibrator was designed to provide information for such corrections. The signal from the onboard calibrator falling on the detectors of each spectrometer, however, has proven to be insufficiently stable to be of use for this correction. Plans for improving the stability of the onboard calibrator signal are discussed in section 5.0 of the paper. The second way of correcting for the signal level output drift of the spectrometer is by referencing the output of each spectrometer to that of the most stable of the spectrometers, spectrometer D. This is done by taking advantage of the spectral overlap between spectrometers. Spectrometer C output can be corrected by adding or subtracting an offset which makes the output of the last three spectral bands equal to the output of the corresponding first three spectral bands of spectrometer D. Similarly, spectrometer B can be corrected relative to C, and spectrometer A can be corrected relative to B, thus limiting the drift of all spectrometers to that of spectrometer $\mathrm{D}$, which is 2.4 percent. This correction is not applied to AVIRIS data during routine radiometric rectification, but the data for making the correction are readily available to those investigators wishing to perform it. Plans for improving the output stability of all four spectrometers are discussed later in the paper.

\subsection{Other factors affecting radiometric calibration accuracy}

Five additional instrument-related factors have been identified which affect the absolute radiometry of AVIRIS. These are: (1) Nonsystematic vibrationally induced fluctuations in instrument output level, (2) polarization sensitivity, (3) spectral calibration uncertainty, (4) spectral stray light, and (5) spatial stray light. The non-systematic vibrational effects were noted earlier in the plots of DN versus crosstrack scan position (Figures 7 and 8 ). They occur randomly in the output of the spectrometers, but at roughly two frequencies: There is a 
signal level fluctuation of as much as 2.0 percent occurring intermittently approximately once per scan cycle. A higher frequency fluctuation is occasionally seen of magnitude 0.7 percent of the signal level with a frequency between 12 and 18 cycles per scan line. These effects do not occur throughout all four spectrometers at the same time. Because of their random nature, they must be included in the calibration error budget. Their nature and eventual removal will be addressed in section 5.0 of the paper.

Polarization sensitivity is measured by placing filters that polarize visible and infrared wavelengths between the AVIRIS foreoptics and the integrating sphere and recording instrument output level as a function of filter polarization angle. A maximum signal loss of 5.0 percent was measured. The effect of spectral calibration uncertainty was calculated by determining the uncertainty of the radiance at the steepest part of the light transfer curve of the integrating sphere, which occurs at $1.4 \mu \mathrm{m}$. An uncertainty of $0.8 \mathrm{~nm}$ in the spectral calibration of spectrometer $C$, which covers this wavelength, yields a radiance uncertainty of 0.5 percent.

Spectral stray light affects radiometric calibration accuracy by contaminating adjacent spectral bands to a degree that is scene-variant. The measurement of spectral stray light is difficult, however, and an adequate technique for characterizing its magnitude in the AVIRIS spectrometers has not yet been devised. A crude measurement has been made by illuminating the instrument with narrow bandwidth light from the laboratory monochromator to establish the signal level on detector elements away from the one illuminated with the monochromator. Within the limits of the instrument electronic noise level, we estimate that the magnitude of the spectral stray light does not exceed 2 percent.

Spatial stray light affects radiometry by scattering light from adjacent pixels of the scene into nearby pixels. It too, is scene-dependent. In an instrument such as AVIRIS, only the foreoptics contribute to spatial stray light. Its magnitude in the AVIRIS foreoptics was measured by illuminating the foreoptics with collimated light passed through a $1 \mathrm{mrad}$ slit. The signal levels were measured across the full field of view of AVIRIS. Measurements were made with the 1 mrad illumination falling on the center of the AVIRIS field of view and at 10 and 20 degrees to either side of the center. The contribution of spatial stray light in the pixels immediately adjacent to the illuminated pixel was 2.0 percent. The signal level at the next pixel was less than 0.3 percent. The 2.0 percent figure was used in the calculation of the overall radiometric accuracy.

\subsection{AVIRIS radiometric calibration accuracy}

The accuracy of the absolute radiometry of AVIRIS was calculated by taking the root sum square of all the elements discussed above and was found to be 7.3 percent. The quantities used in the calculation are summarized in Table 5 . This is the accuracy of the laboratory radiometric calibration of the instrument. Work in progress on the airborne radiometric performance of the instrument using data from the onboard calibrator indicates that the signal levels are less stable in the U-2 than in the laboratory, because the air temperature in the bay of the airplane containing AVIRIS is about 32 degrees F, compared to the 68 degree air temperature in the laboratory. The lower temperature causes the spectrometer heaters to cycle more frequently and for longer periods.

Table 5. Total AVIRIS Radiometric Calibration Error Budget

\begin{tabular}{cc}
\hline Parameter & Magnitude (\%) \\
\hline Integrating Sphere Calibration Accuracy & 3.1 \\
AVIRIS Thermally Induced Output Drift & 2.4 \\
AVIRIS Vibrationally Induced Output Drift & \\
$\quad$ Low Frequency & 2.0 \\
High Frequency & 0.7 \\
Polarization Sensitivity & 5.0 \\
Radiometric Effect of Spectral Calibration & \\
Uncertainty & 0.5 \\
Spectral Stray Light & 2.0 \\
Spatial Stray Light & 2.0 \\
& \\
\hline AVIRIS Radiometric Calibration Accuracy & 7.3 \\
\hline
\end{tabular}

The relative radiometry of the instrument is affected primarily by the accuracy of the spectral calibration of the instrument, for this determines how well the radiance of the integrating sphere at a given wavelength is matched to the corresponding AVIRIS wavelength. Relative radiometry refers to the equalization of detector response so that if, for example, two adjacent detector elements centered at $\lambda_{n}$ and $\lambda_{n+1}$ are illuminated with light at those respective wavelengths but of the same intensity, the output intensity of those detectors after radiometric calibration is equal. Because the output intensity of the integrating sphere varies with wavelength, inaccuracies in the spectral 
calibration can result in inaccuracies in the uniformity correction or relative radiometry. The relative radiometric accuracy can be measured by applying the radiometric calibration to one of the 20 sets of data collected from the integrating sphere and comparing the ratio of the radiance at two adjacent wavelengths from those corrected data to the ratio of the integrating sphere radiance at the same wavelengths as measured by the calibrated spectroradiometer. This was done at several wavelengths. The results of the analysis gave a relative radiometric calibration accuracy of 0.4 percent.

\subsection{FUTURE PLANS}

Further refinements to the laboratory calibration of AVIRIS are planned through additional experiments in calibrating the integrating sphere and through improvements to AVIRIS designed to remove the effects that limit the instrument radiometry. During the 1987-88 winter, the instrument will undergo several improvements at JPL, including a realignment of the foreoptics to remove the vignetting, replacement of the preamplifier and clockdriver boards with more robust versions that are less sensitive to vibration, reconfiguration of the spectrometer heaters with a new thermal control circuit and optimized placement of the heaters to minimize distortion to the spectrometer barrel during the heating cycle, and finally, installation of kinematic mounts between the spectrometers and the instrument mounting rails to reduce the transmission of vibration and warpage from the mounting rails, and reduce the conductive heat loss from the spectrometers to the mounting rails. Also, a stabilized power supply will be installed in the onboard calibrator, and the alignment of the optical fibers from the calibrator to the spectrometers will be improved. Both modifications together should improve the stability of the onboard calibrator signal.

In addition to the work described above, a field calibration experiment was performed in September 1987 to determine the in-flight radiometry of AVIRIS for comparison with the laboratory calibration. Analysis of the data is just beginning at the time of this writing. Additional field experiments are planned in 1988 to verify the performance of AVIRIS and its laboratory calibration after the improvements described above are completed. Results of the next laboratory calibration and the following field calibration experiment will be documented in an AVIRIS project calibration report in 1988.

\subsection{ACKNOWLEDGEMENTS}

The authors would like to express their gratitude to many colleagues for helpful suggestions and stimulating discussions on radiometric calibration which have helped us greatly in developing the procedures described in this paper: At JPL, Jim Conel, Robert Green, Deanne Tucker, Frank Palluconi, Harold Lang, Mike Abrams, John Wellman and Dave Norris; at the University of Arizona, Phil Slater; at the U.S. Department of Agriculture, Ray Jackson; and at the Goddard Space Flight Center, Bruce Guenther.

The work described in this paper was carried out at the Jet Propulsion Laboratory, California Institute of Technology, under a contract with the National Aeronautics and Space Administration.

\subsection{REFERENCES}

1. G. Vane, M. Chrisp, H. Enmark, S. Macenka and J. Solomon, "Airborne visible/infrared imaging spectrometer: an advanced tool for earth remote sensing," Proc. 1984 IEEE Int'l. Geoscience and Remote Sensing Symposium, SP2 15, 751-757 (1984).

2. W. M. Porter and H. T. Enmark, "A system overview of the Airborne Visible/Infrared Imaging Spectrometer (AVIRIS)," Proc. SPIE, 834 (1987).

3. G. Vane and A. F. H. Goetz, "Terrestrial imaging spectroscopy," Remote Sensing of Environment, 24(1), in press (1987).

4. A. F. H. Goetz, G. Vane, J. E. Solomon and B. N. Rock, "Imaging spectrometry for earth remote sensing," Science, 228(4704), 1147-1153 (1985).

5. D. Tucker and G. Vane, "Radiometric calibration of the Airborne Imaging Spectrometer," in Proceedings of the Second Airborne Imaging Spectrometer Data Analysis Workshop, Gregg Vane and Alexander F. H. Goetz, eds., JPL Publication 86-35, Jet Propulsion Laboratory, Pasadena, CA, 17-20 (1986).

6. J. E. Conel, R. O. Green, G. Vane, C. J. Bruegge and R. E. Alley, "AIS-2 radiometry and a comparison of methods for the recovery of ground reflectance," in Proceedings of the Third Airborne Imaging Spectrometer Data Analysis Workshop, Gregg Vane, ed., JPL Publication 87-30, Jet Propulsion Laboratory, Pasadena, CA, 18-47 (1987).

7. V. R. Weidner and J. J. Hsia, "Reflection properties of pressed polytetrafluoroethylene powder," J. Opt. Soc. Am., 71(7), 856-861 (1981).

8. P. N. Slater, personal communication (1986).

9. P. R. Bevington, Data Reduction and Error Analysis for the Physical Sciences, 336 pp., McGraw Hill, New York (1969). 